Research Paper

\title{
Preliminary Study of MR and Fluorescence Dual-mode Imaging: Combined Macrophage-Targeted and Superparamagnetic Polymeric Micelles
}

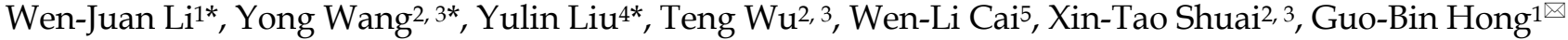 \\ 1. Department of Radiology, the Fifth Affiliated Hospital, Sun Yat-sen University, Zhuhai 519000, China; \\ 2. PCFM Lab of Ministry of Education, School of Materials Science and Engineering, Sun Yat-Sen University, Guangzhou 510275, China; \\ 3. Center of Biomedical Engineering, Zhongshan School of Medicine, Sun Yat-Sen University, Guangzhou 510080, China; \\ 4. Department of Radiology, Hubei Cancer Hospital, Wuhan 430070, China. \\ 5. Department of Radiology, Massachusetts General Hospital and Harvard Medical School, Boston 02114, USA. \\ * These authors contributed equally to this work. \\ $\triangle$ Corresponding author: Guobin Hong, M.D, Department of Radiology, the Fifth Affiliated Hospital, Sun Yat-Sen University, Zhuhai 519000, China; \\ (honggb@mail.sysu.edu.cn) Work Telephone: +86-756-2528666 \\ (C) Ivyspring International Publisher. This is an open access article distributed under the terms of the Creative Commons Attribution (CC BY-NC) license \\ (https://creativecommons.org/licenses/by-nc/4.0/). See http://ivyspring.com/terms for full terms and conditions.
}

Received: 2017.06.24; Accepted: 2017.11.02; Published: 2018.01.01

\begin{abstract}
Purpose: To establish small-sized superparamagnetic polymeric micelles for magnetic resonance and fluorescent dual-modal imaging, we investigated the feasibility of $M R$ imaging (MRI) and macrophage-targeted in vitro.

Methods: A new class of superparamagnetic iron oxide nanoparticles (SPIONs) and Nile red-co-loaded mPEG-Lys3-CA4-NR/SPION polymeric micelles was synthesized to label Raw264.7 cells. The physical characteristics of the polymeric micelles were assessed, the T2 relaxation rate was calculated, and the effect of labeling on the cell viability and cytotoxicity was also determined in vitro. In addition, further evaluation of the application potential of the micelles was conducted via in vitro MRI.

Results: The diameter of the mPEG-Lys3-CA4-NR/SPION polymeric micelles was $33.8 \pm 5.8 \mathrm{~nm}$ on average. Compared with the hydrophilic SPIO, mPEG-Lys3-CA4-NR/SPION micelles increased transversely $(\mathrm{r} 2)$, leading to a notably high $\mathrm{r} 2$ from $1.908 \mu \mathrm{g} / \mathrm{mL}^{-1} \mathrm{~S}^{-1}$ up to $5.032 \mu \mathrm{g} / \mathrm{mL}^{-1} \mathrm{~S}^{-1}$, making the mPEG-Lys3-CA4-NR/SPION micelles a highly sensitive MRI T2 contrast agent, as further demonstrated by in vitro MRI. The results of Confocal Laser Scanning Microscopy (CLSM) and Prussian blue staining of Raw264.7 after incubation with micelle-containing medium indicated that the cellular uptake efficiency is high.

Conclusion: We successfully synthesized dual-modal $M R$ and fluorescence imaging mPEG-Lys3-CA4-NR/SPION polymeric micelles with an ultra-small size and high MRI sensitivity, which were effectively and quickly uptaken into Raw 264.7 cells. mPEG-Lys3-CA4-NR/SPION polymeric micelles might become a new MR lymphography contrast agent, with high effectiveness and high MRI sensitivity.
\end{abstract}

Key words: SPIONs; polymeric micelles; macrophage-targeted; fluorescence imaging; MRI.

\section{Introduction}

The early detection and accurate evaluation of benign and malignant lymph nodes are very important for tumor staging and treatment planning. Lymphadenectomy is considered essential in addition to surgical treatment, and lymph node involvement is also a strong prognostic predictor of patient's outcome $[1,2,3]$. Although the diagnostic value of this conventional technique is limited, MRI is the most effective diagnostic technique for the detection of lymph node metastases. However, the sensitivity and accuracy are relative low due to the detection criteria of lymph node metastases that mainly depend on 
insensitive size and morphology [4,5]. As a result, the normal sized metastatic lymph node is often missed, and it is also difficult to distinguish enlarged inflammatory lymph nodes from metastatic lymph nodes [6]. Considerable effort has been made to solve these problems in recent years. To date, lymphotropic nanoparticle-enhanced MR imaging for lymph node imaging has been given increased attention, and most of the focus has been on superparamagnetic iron oxide (SPIO) $[7,8]$. At the same time, polymeric micelles display many advantages, including a small size, a long half-life, and easy passive targeting. Additionally, as an MR contrast agent, we can obtain polymeric micelles with the property of macrophage targeting by controlling the particle size and superparamagnetism by loading SPIO. Furthermore, we can load Nile red into core micelles to establish small-sized, superparamagnetic, dual-modal polymeric micelles, to evaluate the macrophage uptake efficiency of micelles in vitro, and investigate the feasibility of MRI in vitro.

As a blood pool contrast agent, SPIO can improve the sensitivity and soft-tissue contrast $[9,10$, 11]. In theory, the contrast agent can be administered by two methods in lymph node MR imaging: local injection and intravenous administration. Additionally, the agent particles enter the lymph nodes by two distinct pathways: first, by direct transcapillary passage from high endothelial venules into the medullary sinuses of lymph nodes, followed by engulfment of the particleswithin the lymph nodal parenchyma byphagocytic cells, which is also the major pathway; second, the particles, through nonselective endothelial transcytosis, cross permeable capillaries into the interstitial space, from where the particles drain into the lymph nodes via the lymphatic system; subsequently, the particles are taken up from the interstitium by lymphatic vessels and are transported to regional lymph nodes [12]. Thus far, there is scant published literature about intravenous administration, and most of the literature focused on local injection $[8,13]$. Compared with local injection, intravenous administration has gained increased attention because it enables systemic lymph node imaging, rather than local imaging, by local injection. However, for intravenous administration, the crucial point is that when the diameter of the agent article is great than $40 \mathrm{~nm}$, the agent will be mainly uptaken by the liver and spleen macrophages of the reticuloendothelial system and is rarely absorbed by lymph node macrophages. However, if the size of the agent article is smaller than $40 \mathrm{~nm}$, the situation will be opposite. Based on this situation, in the past several years, lymphotropic nanoparticles loaded with SPIO are a relatively new class of MR contrast agents with unique properties allowing them to be used in a wide variety of clinical applications $[7,14]$. However, there are few studies concerning such small-sized lymphotropic nanoparticles loaded with SPIO.

This study gives full consideration to the new trend in the development of molecular imaging, using nano biotechnology and molecular imaging. By loading hydrophobic SPIO nanoparticles and Nile red into polymeric micelles assembled from the telodendrimer mPEG-b-dendritic oligo-cholic acid (mPEG-Lys3-CA4), we developed superparamagnetic polymeric micelles with a small size (smaller than 40 $\mathrm{nm}$ in diameter) for MR and fluorescent dual-modal imaging to investigate the feasibility of MR imaging and the early detection of occult lymph node metastasis. It is expected to provide a new strategy for the targeted therapy of lymph node metastasis, with great theoretical research significance and clinical potentials.

\section{Materials and Methods}

\section{Materials}

a-Methoxy-ع-hydroxy-poly(ethylene glycol) $(m$ PEG-OH, $\quad \mathrm{nn}=2 \mathrm{kDa})$, Di-tert-butoxycarbonyl-L-lysine (Boc-Lys(Boc)-OH), N-hydroxybenzotriazole (HOBt), 2-(1H-benzotriazole-1-yl)-1,1,3,3tetramethyluronium (HBTU), N,N-diisopropylethylamine (DIPEA) and anhydrous dimethylformamide (DMF) (Sigma-Aldrich) were used as received. Cholic acid (CA) and trifluoracetic acid (TFA) were purchased from J\&K Chemical Technology Co., Ltd. (Beijing, China). Dialysis bags (MWCO: $3.5 \mathrm{kDa}, 14$ $\mathrm{kDa}$ ) were purchased from Shanghai Green Bird Technology Development Co., Ltd., China. Chloroform $\left(\mathrm{CHCl}_{3}\right)$, methanol and diethyl ether were of analytical grade and were purchased from Guangzhou Chemical Reagent Factory, China. $m$ PEG-NH ${ }_{2}$ was synthesized as previously reported [15].

\section{Methods}

\section{Synthesis of the telodendrimer mPEG-b-dendritic oligo-cholic acid (mPEG-Lys $3-\mathrm{CA}_{4}$ )}

The biocompatible amphiphilic telodendrimer was synthesized via solution-phase condensation reactions from $m \mathrm{PEG}_{2 \mathrm{k}}-\mathrm{NH}_{2}$ as previously reported [16]. First, Boc-Lys(Boc)-OH (1.5 equiv) was coupled onto the $\mathrm{N}$-terminal of PEGusing HBTU (1.5 equiv) and HOBt (1.5 equiv) as coupling reagents in DMF overnight. The completion of the reaction was confirmed by the Kaiser test: a yellow color (no blue color) indicates no remaining amino groups. The targeted molecules were precipitated and washed three times with cold diethyl ether. 
Subsequently, the Boc groups were removed by treating with trifluoroacetic acid (TFA) at a polymer concentration of $1 \mathrm{~g} / 10 \mathrm{~mL}$. After stirring for $30 \mathrm{~min}$ at room temperature, the mixture was precipitated into cold diethyl ether, and the precipitate was filtered, washed with diethyl ether, and vacuum-dried to obtain $m$ PEG-Lys. Afterwards, an additional repeat reaction described above was carried out to generate a second generation of dendritic polylysine on one end of PEG ( $m$ PEG-Lys 3 ). Finally, CA molecules (6.0 equiv) were coupled to the $\mathrm{N}$-terminal of PBLA-Lys 3 via an amidation reaction with HBTU (6.0 equiv) and HOBt (6.0 equiv) as coupling reagents. The reaction was proceeded in DMF overnight and then was precipitated and washed by cold methanol followed by filtering and vacuum-drying to finally obtain $m$ PEG-Lys $3-\mathrm{CA}_{4}(\mathrm{Mn}$ $=3.9 \mathrm{kDa}$, calculated from the ${ }^{1} \mathrm{H}$ NMR spectrum).

\section{Synthesis of hydrophobic $\mathrm{Fe}_{3} \mathrm{O}_{4}$ nanoparticles}

The T2contrast of hydrophobic $\mathrm{Fe}_{3} \mathrm{O}_{4}$ nanoparticles - that is, superparamagnetic iron oxide nanoparticles (SPIONs) - with the diameter of 4-6 nm were synthesized as previously reported [17]. Briefly, iron(III) acetylacetonate ( $2 \mathrm{mmol}), 1,2$-hexadecanediol $(10 \mathrm{mmol})$, oleic acid $(6 \mathrm{mmol})$ and oleylamine (6 $\mathrm{mmol}$ ) were dissolved in $20 \mathrm{~mL}$ of benzyl ether in a reaction flask with magnetic stirring under argon. Next, the mixture was heated to $200{ }^{\circ} \mathrm{C}$, kept for $2 \mathrm{~h}$, and finally refluxed at $300{ }^{\circ} \mathrm{C}$ for an additional $1 \mathrm{~h}$. Subsequently, the black solution was cooled to room temperature under the protection of argon, precipitated into ethanol $(200 \mathrm{~mL})$ and then centrifuged (6000 rpm, $3 \mathrm{~min})$ to collect the precipitate. The obtained products were dissolved in $20 \mathrm{~mL}$ of hexane, centrifuged (12000 rpm, $6 \mathrm{~min}$ ) to remove large aggregations, and precipitated into ethanol $(200 \mathrm{~mL})$ for another time. Finally, the black-brown nanoparticles were redispersed into hexane and stored at $4{ }^{\circ} \mathrm{C}$.

\section{Preparation of Nile red/SPIO co-loaded Michelle (mPEG-Lys $3-\mathrm{CA}_{4}-\mathrm{NR} / \mathrm{SPIONs}$ )}

To prepare the SPIONs and Nile red co-loaded micelles, $1 \mathrm{mg}$ of superparamagnetic iron oxide (SPIO), $0.2 \mathrm{mg}$ of Nile red and $20 \mathrm{mg}$ of polymer (PEG-Lys3-CA4) were co-dissolved in $2 \mathrm{~mL}$ of dimethyl sulfoxide (DMSO) and chloroform ( $\mathrm{v}: \mathrm{v}=$ 1:3). Under sonication (VCX130, Sonics, USA, $20 \mathrm{kHz}$, $40 \%$ power level), the above solution was added dropwise $20 \mathrm{~mL}$ of phosphate-buffered saline (PBS). After the organic solvent chloroform was removed by rotary evaporation, the solution was filtered through a syringe filter (pore size: $450 \mathrm{~nm}$ ) to eliminate free SPIO, Nile red and large aggregates, followed by ultrafiltration using a MILLIPORE centrifugal filter device (MW cutoff: $100 \mathrm{kDa}$ ) to remove DMSO and other hydrophilic impurities. In the meantime, we also prepared Nile red-loaded micelles-that is, mPEG-Lys3-CA4-NR micelles - in the same way.

\section{H NMR spectra measurements}

${ }^{1} \mathrm{H}$ NMR spectra were carried out to confirm the synthesis of the designed telodendrimer mPEG-bdendritic oligo-cholic acid using a Varian Unity 300 $\mathrm{MHz}$ spectrometer and $\mathrm{CDCl}_{3}-d$ or DMSO- $d_{6}$ as the solvent at room temperature.

\section{Dynamic light scattering (DLS) measurements}

The sizes and zeta potentials of mPEG-Lys3CA4-NR/SPION micelles were measured using dynamic light scattering (DLS). The measurements of the particle size and zeta potential were carried out using90 Plus/BI-MAS equipment (Brookhaven Instruments Corporation, USA) at $25{ }^{\circ} \mathrm{C}$. Additionally, a standard electrophoresis mini-cell from Brookhaven was used for the measurement of zeta potentials. The data of particle size and zeta potential were collected using an auto-correlator with detection angles of scattered light at $90^{\circ}$ and $15^{\circ}$, respectively. For each sample, the data were represented as the mean \pm standard deviation (SD) of five measurements.

\section{Transmission electron microscopy (TEM) measurements}

TEM imaging was obtained at room temperature using a Hitachi model H-7650 TEM operated at $80 \mathrm{kV}$ to determine the morphology characteristics of mPEG-Lys3-CA4-NR/SPION micelles. Samples were prepared by drying a drop $(5 \mu \mathrm{L}, 0.5 \mathrm{mg} / \mathrm{mL})$ of the sample solution on a copper grid coated with amorphous carbon, followed by blotting with filter paper after $1 \mathrm{~h}$. For the negative staining of samples, $10 \mu \mathrm{L}$ of uranyl acetate solution ( $2 \mathrm{wt} \%$ in water) was added to the copper grid; after $1 \mathrm{~min}$, the grid was blotted with a piece of filter paper. The grid was finally dried overnight at room temperature inside a desiccator before TEM observation.

\section{Measurement of SPIO loading and the Nile red content}

The iron and Nile red content of the micelles was determined by atomic absorption spectrometry (ASS, Z-200, Hitachi, Japan) and fluorescence spectroscopy (PE-LS55; PerkinElmer Ltd., United Kingdom), respectively. Briefly, before $\mathrm{mPEG}^{-\mathrm{Lys}_{3}-\mathrm{CA}_{4}-}$ NR/SPIONs were suspended in $1 \mathrm{M} \mathrm{HCl}$ solution to allow for polymer degradation and complete dissolution, it was first weighed, and then the iron concentration was determined at a specific 
Fe-absorption wavelength $(248.3 \mathrm{~nm})$ based on a previously established calibration curve. The SPIO loading density was calculated as the ratio of iron oxide over the total weight of mPEG-Lys $3_{3}$ $\mathrm{CA}_{4}$-NR/SPIONs.

\section{$\mathrm{T} 2$ relaxivities of Nile red/SPIO-co-loaded micelles and hydrophilic SPIO}

Magnetization measurements were performed using a clinical 3.0-T MRI scanner (GE compony Discovery MR750) with an 8 circular head coil at room temperature. Fast spin echo (FSE) T2-weighted images (T2WI) and T2-mapping were acquired, and T2- mapping was also acquired using single section multi-spin-echo sequences. The detailed acquisition parameters of T2-weighted images were as follows: $\mathrm{TR} / \mathrm{TE}=5000 / 111 \mathrm{~ms}, \mathrm{FOV}=100 \mathrm{~mm}$, matrix of $256 * 256$, section thickness of $2 \mathrm{~mm}$, and region of interest (ROI) of $28 \mathrm{~mm}^{2}$. A ROI was selected in each sample, and the T2 relaxation times were obtained. MR imaging was achieved using Nile red/SPIO co-loaded micelles (mPEG- Lys3-CA4-NR/SPIONs micelles) and hydrophilic SPIO both with different Fe3+ concentrations of $0,0.5,1,2$, and $4 \mu \mathrm{g} / \mathrm{mL}$. The transversal relaxation times (T2) of the SPIO polymer and hydrophilic SPIO using phosphate-buffered saline (PBS) as solvent were measured using MRI, and we evaluated the MRI sensitivity as assessed by the measurement of $\mathrm{T} 2$ relaxivities. The $\mathrm{T} 2$ relaxivities of the SPIO polymer and hydrophilic SPIO were calculated from the slope of the linear plots of the $\mathrm{r} 2$ relaxation rates $(1 / \mathrm{T} 2)$ versus Fe concentration. The increase in the $\mathrm{r} 2$ relaxation rates $(1 / \mathrm{T} 2)$ with increasing $\mathrm{Fe} 3+$ concentration was analyzed by the linear least squares regression analysis.

\section{Cell Preparation}

Raw264.7 cells (mouse macrophage cell line) were obtained from Cyagen Bioscience Technology Co. (Guangzhou, China) and were cultured in Dulbecco's Modified Eagle's Medium (DMEM; Gibco, New York, NY, USA) containing 10\% fetal bovine serum (FBS; Gibco, New York, NY, USA), 1\% penicillin $(100 \mathrm{U} / \mathrm{mL})$, and streptomycin $(100 \mathrm{U} / \mathrm{mL})$. Raw264.7 cells were cultured at $37^{\circ} \mathrm{C}$ in a humidified $5 \% \mathrm{CO}_{2}$ atmosphere.

\section{In vitro cytotoxicity test}

The cytotoxicity of mPEG-Lys 3 -CA $\mathrm{CA}_{4}-\mathrm{NR} / \mathrm{SPION}$ micelles and mPEG-Lys ${ }_{3}-\mathrm{CA}_{4}-\mathrm{NR}$ micelles was investigated using the methylthiazolyldiphenyl-tetrazolium bromide (MTT) cell proliferation assay. Approximately 10,000 Raw264.7 cells were seeded into each well of the 96-well plates and were cultured at $37{ }^{\circ} \mathrm{C}$ in a humidified $5 \% \mathrm{CO}_{2}$ atmosphere for $6 \mathrm{~h}$. Next, Raw264.7 cells were incubated for $36 \mathrm{~h}$ in a humidified atmosphere containing $5 \% \mathrm{CO}_{2}$ in culture medium supplemented with a series of concentrations of mPEG-Lys $3-\mathrm{CA}_{4-}$ NR/SPION micelles and mPEG-Lys $3-\mathrm{CA}_{4}-\mathrm{NR}$ micelles; the final concentrations of iron in the mPEG-Lys ${ }_{3}-\mathrm{CA}_{4}$ - NR/SPION micelleswere $0,5,10,20$, 40, 80, $160 \mu \mathrm{g} / \mathrm{mL}$ (the mPEG-Lys ${ }_{3}-\mathrm{CA}_{4}$-NR/SPION and mPEG-Lys 3 -CA $\mathrm{CA}_{4}-\mathrm{NR}$ micelle concentrations were both 0, 108.8, 217.5, 435, 870, 1740, and 3480 $\mu \mathrm{g} / \mathrm{mL}$ ).Next, MTT reagent (Sigma, $0.5 \% ; 20 \mu \mathrm{l}$ per well) was added, followed by incubation for $4 \mathrm{~h}$. The medium was discarded, and $150 \mathrm{~mL}$ of dimethyl sulfoxide (DMSO) was added to each well. After shocking for $15 \mathrm{~min}$ with a shaking table, the absorbance at $570 \mathrm{~nm}$ was recorded using a microplate reader (SpectraMaxM5; Molecular Devices, CA, USA). Cell viability was determined by the following equation: Cell viability $(\%)^{1 / 4}(\mathrm{Ni} / \mathrm{Nc})$ 100, where $\mathrm{Ni}$ and $\mathrm{Nc}$ are the absorbances of surviving cells treated with and without PEG-Lys 3 -CA ${ }_{4}$-SPIONs micelles, respectively.

\section{Confocal laser scanning microscopy (CLSM)}

Raw264.7 cells were inoculated into Petri dishes at a density of 50,000 cells per dish for $6 \mathrm{~h}$. Next, the medium was discarded, and $2 \mathrm{~mL}$ of culture medium was added containing mPEG-Lys 3 -CA $\mathrm{CA}_{4}-\mathrm{NR} / \mathrm{SPION}$ micelles at a Nile red concentration of $1 \mu \mathrm{g} / \mathrm{mL}$, followed by incubation at $37^{\circ} \mathrm{C}$ in a humidified $5 \%$ $\mathrm{CO}_{2}$ atmosphere at $0.5 \mathrm{~h}, 1 \mathrm{~h}, 2 \mathrm{~h}, 4 \mathrm{~h}, 6 \mathrm{~h}$, and $8 \mathrm{~h}$. Thereafter, the cells were washed three times with phosphate-buffered saline (PBS) and then were fixed with $4 \%$ glutaraldehyde for approximately $15 \mathrm{~min}$, followed by washing the cells again, and nuclei were stained blue with DAPI $(10 \mu \mathrm{g} / \mathrm{mL})$ for approximately $2 \mathrm{~min}$. The cells for microscopic observation using a confocal laser scanning microscope (FV1000; OLYMPUS, Japan) to identify the micelles inside cells. Nile red was excited at $485 \mathrm{~nm}$ with an emission at 595 $\mathrm{nm}$. Images were processed using the IBM Graphics workstation.

\section{Prussian blue staining}

Approximately 50,000 Raw264.7 cells were seeded into each well of 6-well plates, and two groups were designed: the time group and concentration group. The time group was incubated for $2 \mathrm{~h}, 4 \mathrm{~h}, 6 \mathrm{~h}$ and included mPEG-Lys $3-\mathrm{CA}_{4}$-NR/SPION micelles with an iron concentration of $40 \mu \mathrm{g} / \mathrm{mL}$; the concentration group was incubated for $6 \mathrm{~h}$ and included mPEG-Lys 3 -CA $-\mathrm{CA}_{4}-\mathrm{NR} / \mathrm{SPION}$ micelles with some iron concentrations of 10,20 , and $40 \mu \mathrm{g} / \mathrm{mL}$. For each group, they were all incubated in a humidified atmosphere containing $5 \% \mathrm{CO}_{2}$ in culture medium at $37^{\circ} \mathrm{C}$. Subsequently, Raw 264.7 cells were washed 
three times with phosphate-buffered saline (PBS) and then were fixed with $4 \%$ glutaraldehyde for approximately $15 \mathrm{~min}$. The medium was discarded, and then $2 \mathrm{~mL}$ of Prussian blue solution (1\% hydrochloride: $1 \%$ potassium ferrocyanide (II) trihydrate $=1: 1$ ) was added, followed by incubation for $30 \mathrm{~min}$ and washing of the Raw264.7 cells with phosphate buffered saline (PBS). The cells were washed three times again with PBS, and iron staining was subsequently observed using an inverted optical microscope.

\section{In vitro $\mathrm{MR}$ imaging}

For in vitro MR imaging, $5 \times 10^{6}$ Raw264.7 cells were seeded into each well of the 6-well plates. The concentration group was incubated in culture medium that included mPEG-Lys3-CA4- NR/SPION micelles with different iron concentrations of $0,5,10$, $20,40 \mu \mathrm{g} / \mathrm{mL}$ for $6 \mathrm{~h}$, and the time group was inoculated in culture medium that included mPEGLys3- CA4- NR/SPION micelles with different iron concentrations of $40 \mu \mathrm{g} / \mathrm{mL}$ for $0 \mathrm{~h}, 0.5 \mathrm{~h}, 2 \mathrm{~h}, 4 \mathrm{~h}, 6 \mathrm{~h}$, and $8 \mathrm{~h}$. Both groups were incubated at $37^{\circ} \mathrm{C}$ in a humidified atmosphere containing $5 \% \quad \mathrm{CO}_{2}$. The labeled cells were re-suspended in $500 \mu \mathrm{l}$ of $0.5 \%$ agarose gel (Invitrogen, Merelbeke, Belgium) and then were transferred into EP $(200 \mu \mathrm{l})$ tubes. In vitro MRI measurements were performed using a clinical 3.0 T MRI scanner (GE company Discovery MR750) with an 8 circular head coil at room temperature. Fast spin echo (FSE) T2-weighted images (T2WI) and T2-mapping were acquired, and T2- mapping also used single section multi-spin-echo sequences. The detailed acquisition parameters of T2-weighted images were as follows: TR/TE $=5000 / 111 \mathrm{~ms}$; FOV=100 mm; Matrix: 256*256; section thickness: 2 $\mathrm{mm}, \mathrm{ROI}=28 \mathrm{~mm}^{2}$. T2-maps were acquired using the following parameters: TR=5000; $\mathrm{TE}=6.4 、 12.8 、 19.1$ 、25.5、31.9、38.3、44.7、 and 51.0 s; Matrix: 256*256; section thickness: $2 \mathrm{~mm}, \mathrm{ROI}=28 \mathrm{~mm}^{2}$. One ROI was selected in each sample, and the values of T2 relaxation times were obtained.

\section{Statistical Analyses}

The T2 relaxivities and viability assay results were compared using unpaired Student $t$ test. One-way analysis of variance was used to calculated the change in the T2 signal. $P$ values $<0.05$ were considered to indicate statistical significance. All calculations were performed using Statistical Product, Service Solutions (SPSS) software (Version 21), GraphPad Prism 6 software and Image-Pro Plus 6.0.

\section{Results and Discussion}

\section{Preparation and characterization of mPEG-Lys 3 -CA $-A_{4}$-NR/SPION nanoparticles}

The biocompatible amphiphilic telodendrimer (mPEG-Lys3-CA4) was synthesized via solution-phase condensation reactions from $m \mathrm{PEG}_{2 \mathrm{k}}-\mathrm{NH}_{2}$ as previously reported [16] [See: J. T. Luo, K. Xiao, Y. P. Li, J. S. Lee, L. F. Shi, Y. H. Tan, L. Xing, R. H. Cheng, G. Y. Liu, K. S. Lam, Bioconjugate Chem. 2010, 21, 1216-1224]. It was synthesized by multistep chemical reactions as shown in Figure 1. Previous studies have shown that the PEG covering the SPIONs could increase the bio-stability [18].

Figure 2 shows the ${ }^{1} \mathrm{H}$ NMR spectra of (i) $m$ PEG-Lys(Boc) ${ }_{2}$, (ii $m$ PEG-Lys, (iii) $m$ PEG-Lys $3-(B o c)_{4}$ and (iv) $m$ PEG-Lys 3 in $\mathrm{CDCl}_{3}$. The major resonance peaks of the copolymer in the ${ }^{1} \mathrm{H}$ spectra fit well into the expected chemical structure: $3.38 \mathrm{ppm}\left(\mathrm{s},-\mathrm{OCH}_{3}\right.$ of PEG, a), 3.45-3.76 ppm (m, $-\mathrm{CH}_{2} \mathrm{CH}_{2} \mathrm{O}$ of PEG, b), 1.47-2.08 ppm (m, - $\mathrm{CHCH}_{2} \mathrm{CH}_{2} \mathrm{CH}_{2} \mathrm{CH}_{2} \mathrm{NH}$ - of lys, c, d and e), $3.10 \mathrm{ppm}\left(\mathrm{m},-\mathrm{CHCH}_{2} \mathrm{CH}_{2} \mathrm{CH}_{2} \mathrm{CH}_{2} \mathrm{NH}\right.$ - of lys, f), $1.42 \mathrm{ppm}\left(\mathrm{s},-\mathrm{CH}_{3}\right.$ of Boc, g). The presence and absence of characteristic shifts of protons in the Boc group indicated the success of reactions coupling Boc-Lys(Boc)-OH and removing the Boc group, respectively. The grafting efficiency of Boc-Lys(Boc)-OH in $m$ PEG-Lys(Boc) $)_{2}$ and $m$ PEG-Lys 3 -(Boc) 4 were $95 \%$ and $89 \%$, respectivelyper the integral area ratio of protons from the Boc group and methylene group in PEG.

Figure 3 indicates that the major CA proton shifts appeared at $0.56,0.80$ and $0.91 \mathrm{ppm}$, demonstrating the successful synthesis of CA. Figure 4 reveals the GPC curves of $m$ PEG-Lys 3 and $m$ PEG-Lys $3-\mathrm{CA}_{4}$ in THF at a flow rate of $1 \mathrm{~mL} / \mathrm{min}$. The two polymers showed a unimodal molecular weight distribution in their GPC chromatograms, and the final polymer showed an obviously higher molecular weight than the prepolymer, indicating that the CA molecules were coupled onto $m$ PEG-Lys 3 successfully.

All results demonstrated that the synthesis of mPEG-Lys3-CA4 was successful, and GPC and $1 \mathrm{H}$ NMR measurements indicated that the copolymers with a desirable molecular weight and polymer dispersity were synthesized via multi-step chemical reactions. The molecular weight and polymer dispersity index (PDI) of $m$ PEG-Lys 3 and $m$ PEG-Lys $3-\mathrm{CA}_{4}$ are shown in table 1 . The polymer dispersity index of $m$ PEG-Lys 3 is 1.04, which is smaller than that of $m$ PEG-Lys $3-\mathrm{CA}_{4}$ (1.07). 


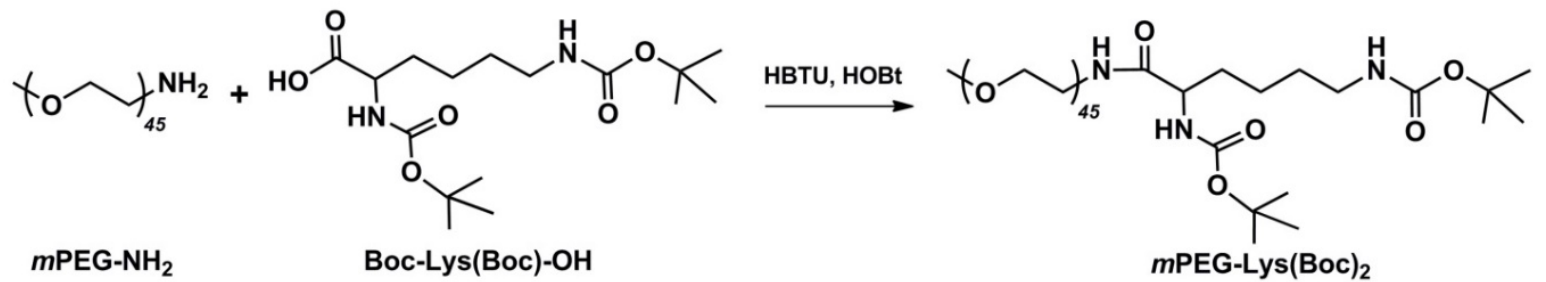

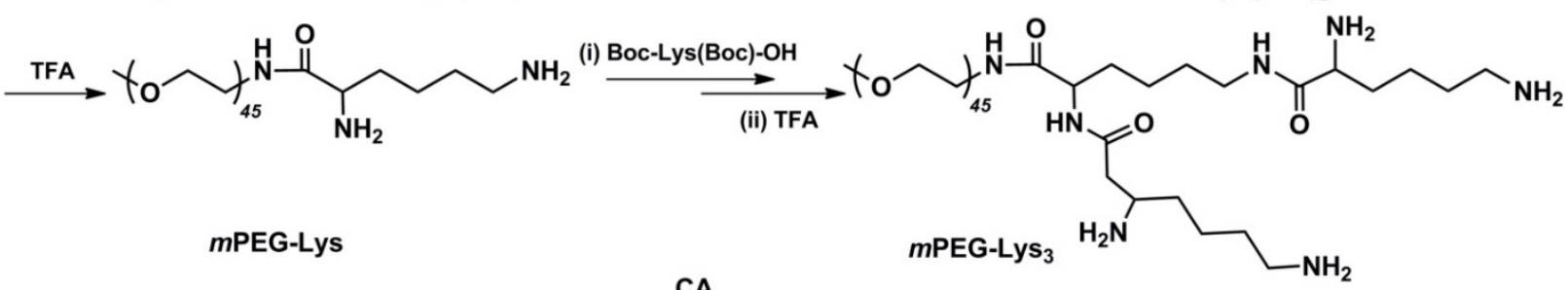

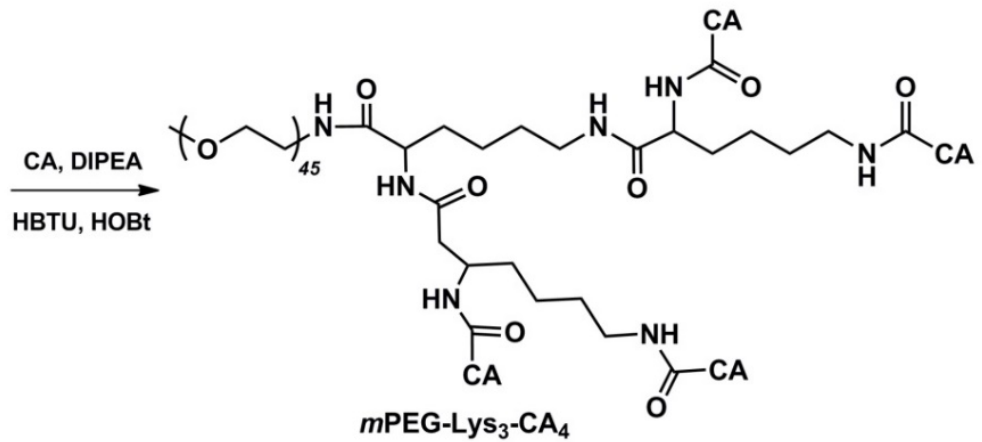

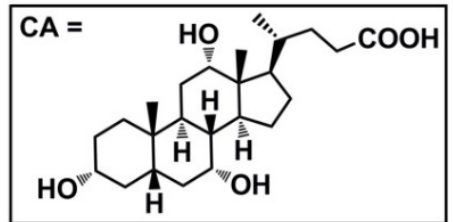

Figure 1. Synthetic approach for the polymer mPEG-Lys3 $-\mathrm{CA}_{4}$

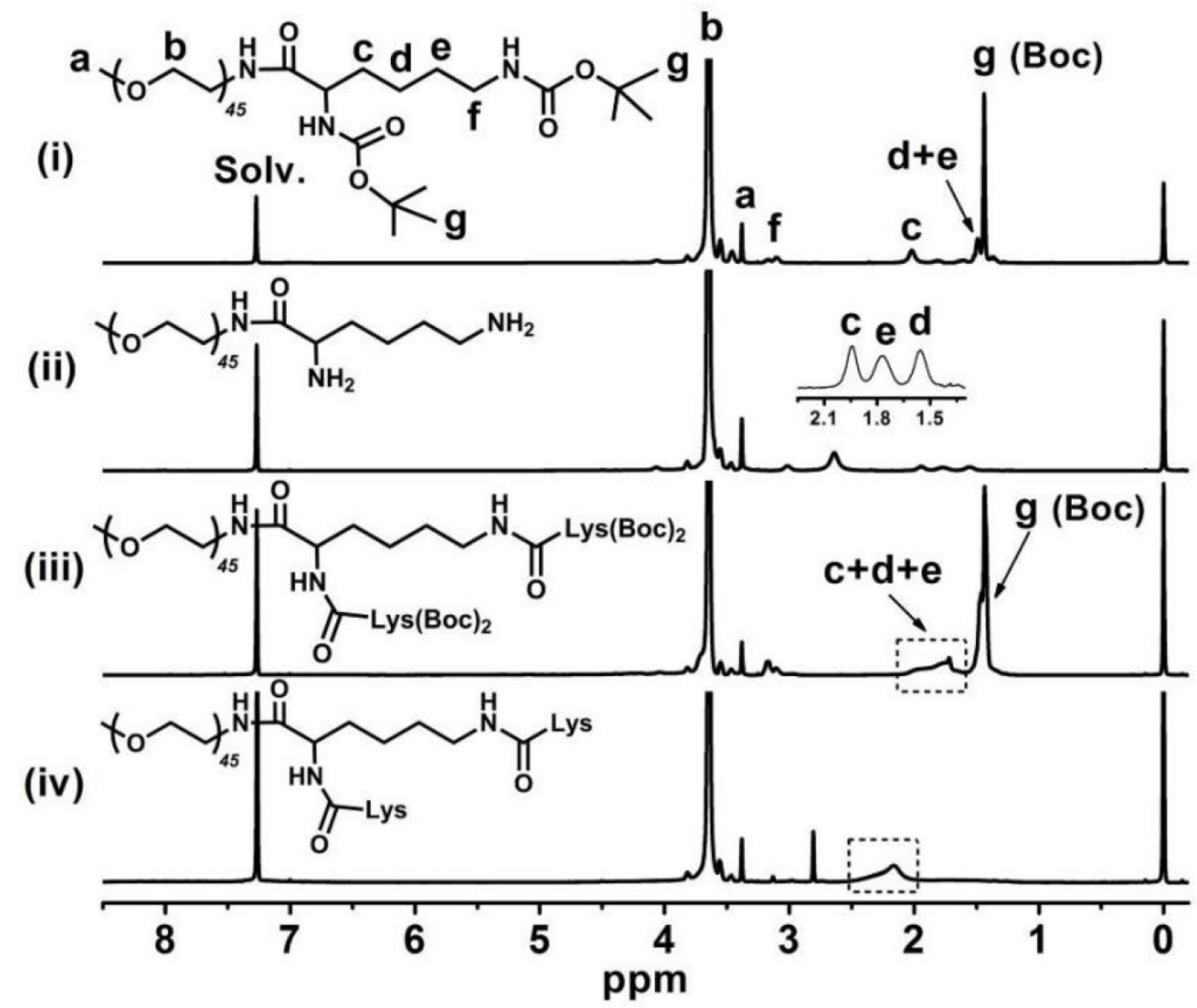

Figure 2. $1 \mathrm{H}$ NMR spectra of (i) mPEG-Lys(Boc)2, (ii) mPEG-Lys, (iii) mPEG-Lys 3 -(Boc) 4 and (iv) mPEG-Lys 3 in $\mathrm{CDCl}_{3}-d$. 


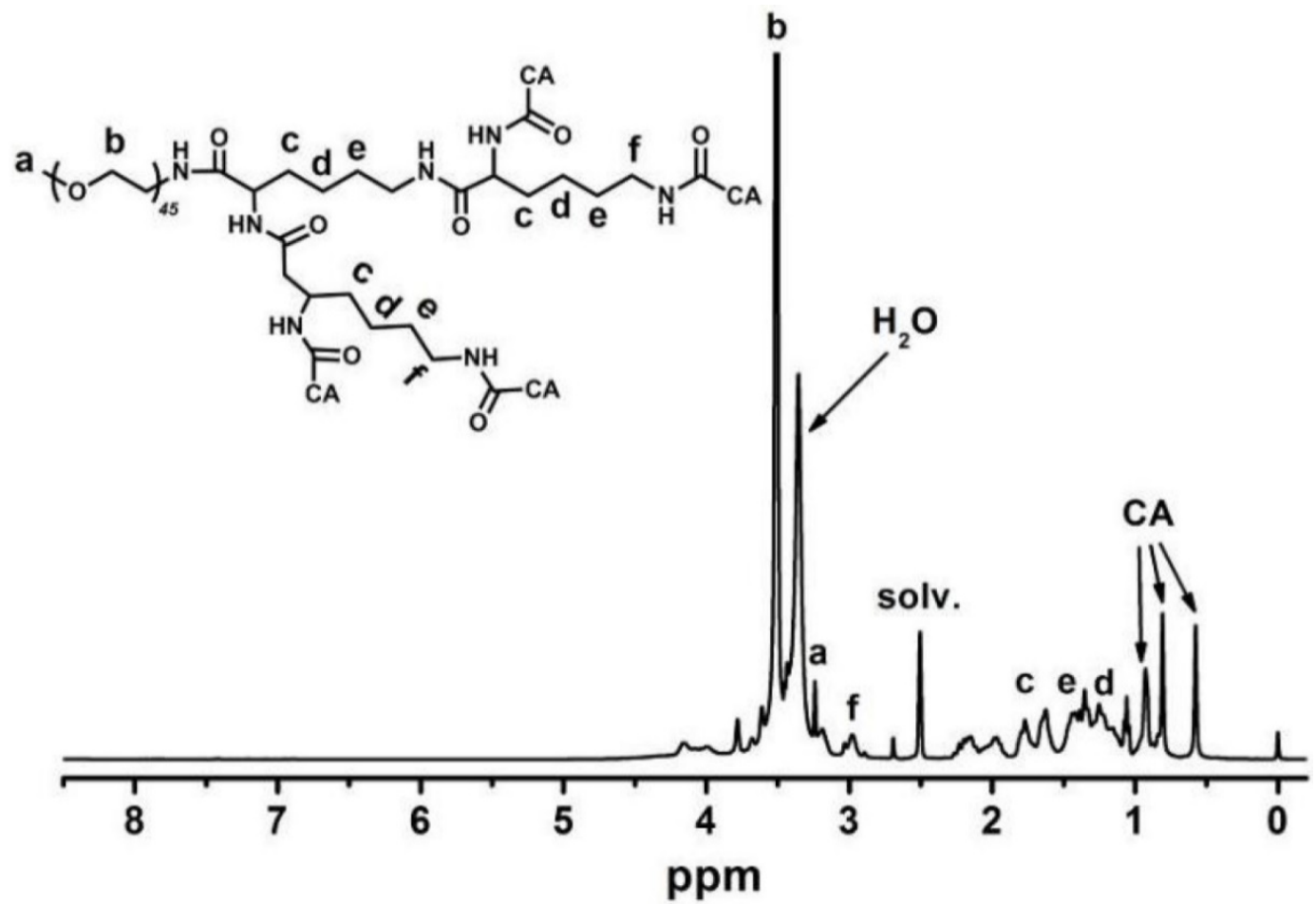

Figure 3. $1 \mathrm{H} N M R$ spectrum of $m P E G-\mathrm{Lys}_{3}-\mathrm{CA}_{4}$ in DMSO- $\mathrm{d}_{6}$. After cholic acid (CA) reacted onto $\mathrm{mPEG}-\mathrm{Lys} 3$, the major CA proton shifts appeared at $0.56,0.80$ and $0.91 \mathrm{ppm}$.

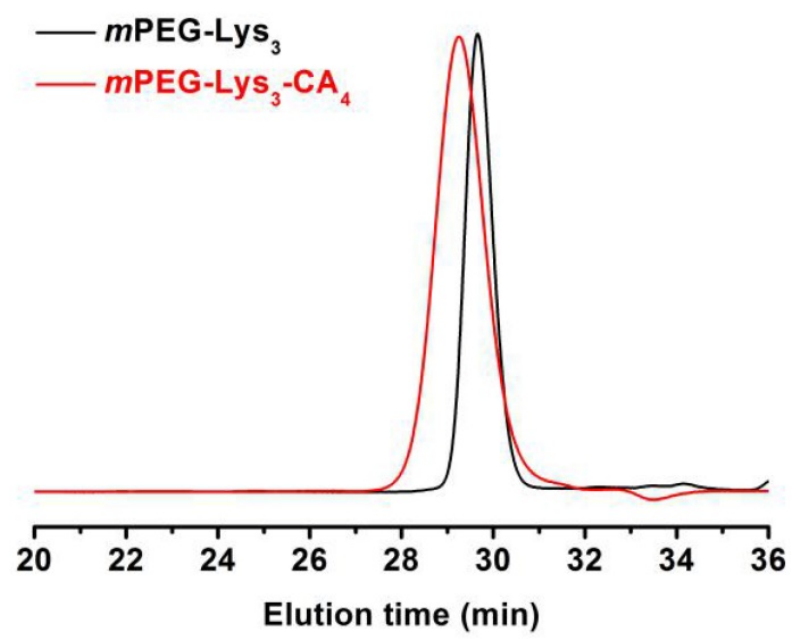

Figure 4. GPC curves of $m$ PEG-Lys $s_{3}$ and $m P E G-L y s_{3}-C_{4}$ in THF at a flow rate of $1 \mathrm{~mL} / \mathrm{min}$.

Table 1. Molecular weight and polymer dispersity index (PDI) of $m$ PEG-Lys3 and $m$ PEG-Lys3-CA4.

\begin{tabular}{llll}
\hline Polymer & $M_{\mathrm{n}^{\mathrm{a}}}$ & $M_{\mathrm{n}^{\mathrm{b}}}$ & $M_{\mathrm{w}} / M_{\mathrm{n}} \mathrm{b}^{\mathrm{b}}$ \\
\hline$m$ PEG-Lys3 & 2500 & 3100 & 1.04 \\
$m$ PEG-Lys3-CA 4 & 3900 & 4900 & 1.07 \\
\hline
\end{tabular}

a calculated by ${ }^{1} \mathrm{H}$ NMR; ${ }^{b}$ measured by GPC.

The T2 contrast of hydrophobic $\mathrm{Fe}_{3} \mathrm{O}_{4}$ nanoparticles with the diameter of 4-6 nm was synthesized as previously reported [17]. Briefly, a simple but effective method, the high-temperature decomposition method, was adopted to obtain hydrophobic $\mathrm{Fe}_{3} \mathrm{O}_{4}$ nanoparticles, and this method can make them water soluble and biocompatible. Figure 5 describes the telodendrimer mPEG-bdendritic oligo-cholic acid (mPEG-Lys3-CA4) being introduced to encapsulate the SPIONs and Nile red. The $\mathrm{Fe}_{3} \mathrm{O}_{4}$ nanoparticle size was approximately 4-6 $\mathrm{nm}$. Dynamic light scattering was adopted to analyze and determine the diameters of PEG-Lys3-CA4-NR and mPEG-Lys3-CA4-NR/SPIO micelles, and the results are shown in Figure 6, which shows the size and size distribution of mPEG-Lys3-CA4-NR micelles and mPEG-Lys3-CA4-NR/SPIO micelles. The hydrodynamic diameter of mPEG-Lys3-CA4-NR micelles was $24.2 \pm 3.3 \mathrm{~nm}$, which was close to the diameter of blank micelles $(21.8 \pm 1.9 \mathrm{~nm}$, data not shown in the figure). However, when SPIO was loaded into the micelle, the data came to $33.8 \pm 5.8 \mathrm{~nm}$. Since dynamic light scattering measurement provides information about the size of particles, the increase in the particle diameter upon micellar encapsulation apparently could be attributed to the hydrodynamic radius of the polymeric coating on the iron oxide nanoparticles. The hydrodynamic size of nanoparticles in physiological fluids is very important because it is known to significantly affect not only their plasma half-life time but also their biodistribution and pharmacokinetic properties. A 
previous study noted that the capillary diameter of the reticuloendothelial system is approximately $50 \mathrm{~nm}$ [19]; in our study, the micelle diameter was smaller than $40 \mathrm{~nm}$, and the small appropriate sizes made the micelles target lymphaden for effective diagnosis. Magnetic nanoparticles for MR signal enhancement must be well defined in structure and size because the size can affect the MR signals. In our research, as shown in Figure 7, transmission electronic microscopy (TEM) observation of both mPEG-Lys3-CA4-NR micelles and mPEG-Lys3-CA4- NR/SPIO micelles and the images indicated that both types of micelles were spherically shaped with a uniform size, which was in line with that detected by DLS (Scale bars $=50$ $\mathrm{nm}$ ) and the encapsulation of clustered SPIONs inside the inner aqueous core of the micelles. The loading contents of SPIO and Nile red ofmPEG-Lys3-CA4-NR/SPION micelles were $4.4 \%$ and $0.9 \%$, respectively, and the zeta potential of mPEG-Lys3-CA4-NR/SPION micelles was $-0.01 \pm 3.5$ as shown in table 2.

\section{Safety and effectiveness of labeling}

The cytotoxicity of the mPEG-Lys3-CA4-NR/ SPION micelles and mPEG-Lys3-CA4-NR micelles was further revealed using the MTT cytotoxicity assay in Raw 264.7 cells. As shown in Figure 8, the Nile red-loaded micelles did not show obvious cell growth inhibition even at very high concentrations of micelles, reaching 3,500 $\mu \mathrm{g} / \mathrm{mL}$. When coated with SPION, the cytotoxicity was increased, but the Nile red/SPION co-loaded micelles did not affect the cell viability of RAW 264.7 cells at the highest concentration of $875 \mu \mathrm{g} / \mathrm{mL}$ (the iron concentration was $40 \mu \mathrm{g} / \mathrm{mL}$ ), and mPEG-Lys3-CA4-NR/SPIO micelles negatively affected the Raw 264.7 cell viability at iron concentrations from 40 to $180 \mu \mathrm{g} / \mathrm{mL}$ in a dose-dependent manner - that is, the cytotoxicity will increase with the increase in $\mathrm{Fe} 3+$ concentration. Although the cytotoxicity of mPEG-Lys3-CA4-NR/ SPION micelles was slightly high, we have indicated that $40 \mu \mathrm{g} / \mathrm{mL}$ is sufficient for magnetic resonance imaging.

Table 2. SPIO and Nile red contents of mPEG-Lys3-CA4-NR/SPION micelles, and the sizes and zeta potentials of micelles were determined by DLS.

\begin{tabular}{lllll}
\hline micelles & $\begin{array}{l}\text { SPIO } \\
\text { loading }(\%)\end{array}$ & $\begin{array}{l}\text { Nile red } \\
\text { loading }(\%)\end{array}$ & $\begin{array}{l}\text { Size } \\
(\mathrm{nm})\end{array}$ & $\begin{array}{l}\text { Zata } \\
(\mathrm{mV})\end{array}$ \\
\hline mPEG-Lys3-CA4-NR/SPIONs & $4.4 \pm 0.2$ & $0.9 \pm 0.1$ & $33.8 \pm$ & $-0.01 \pm 3.5$ \\
& & & 5.8 & \\
mPEG-Lys3-CA4-NR & & & $24.2 \pm 3.3$ & $0.11 \pm 2.2$
\end{tabular}

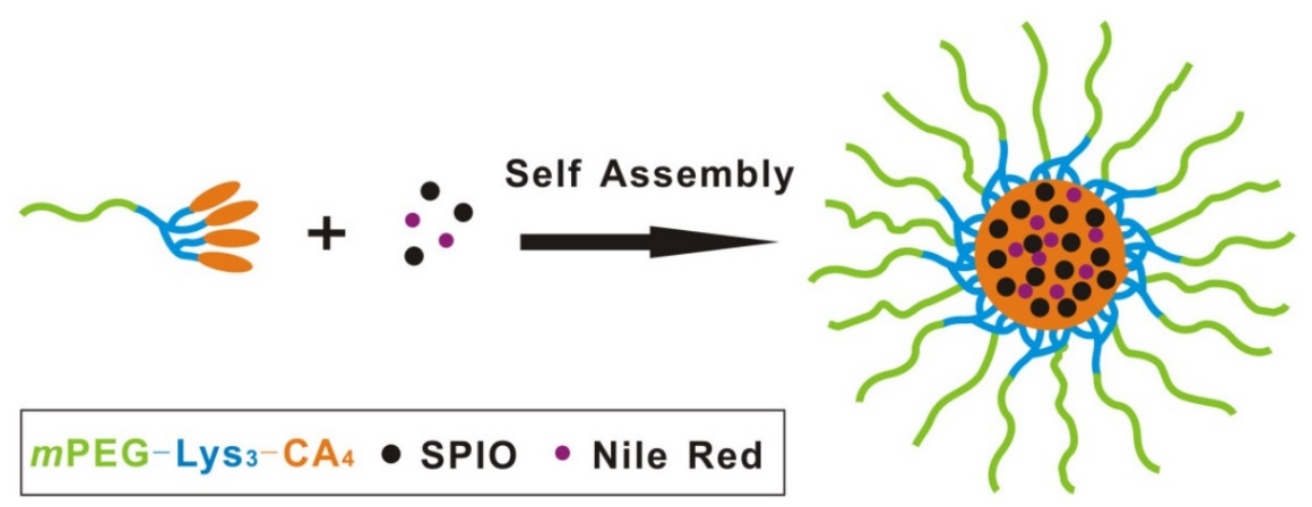

Figure 5. Formation of SPIO and mPEG-Lys3-CA4-NR/SPION micelles for MR and optical imaging.
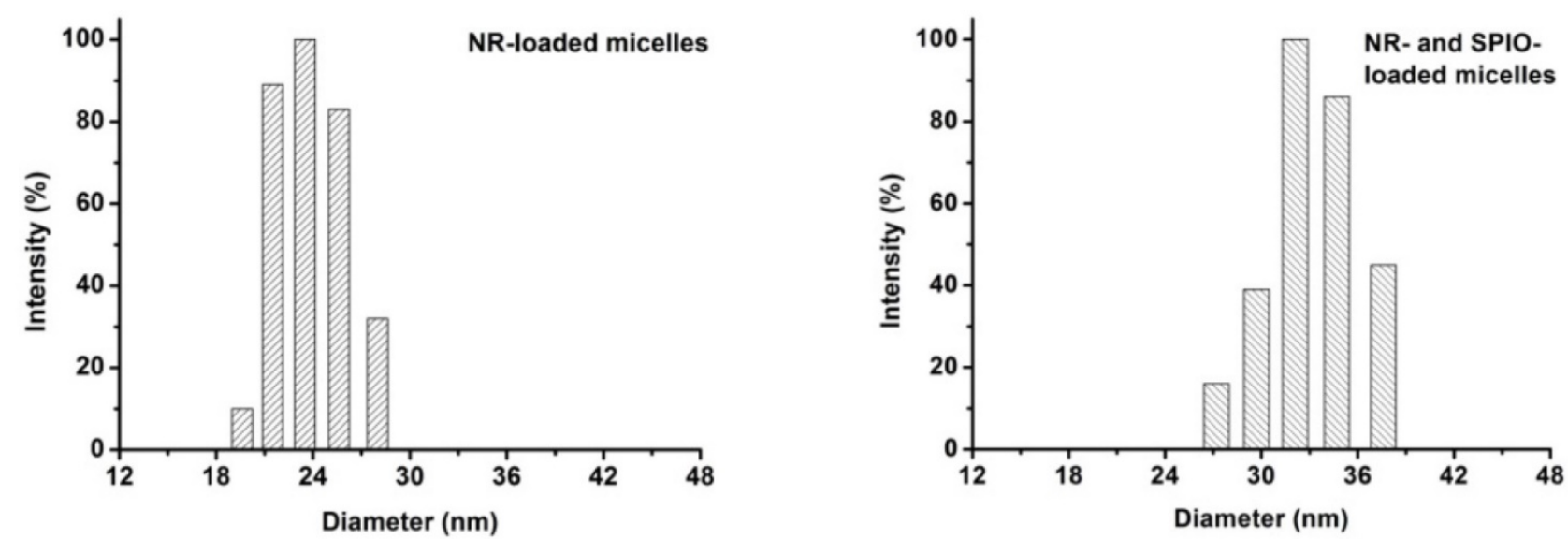

Figure 6. The size and distribution of mPEG-Lys3-CA4-NR micelles and mPEG-Lys3-CA4-NR/SPION micelles. 

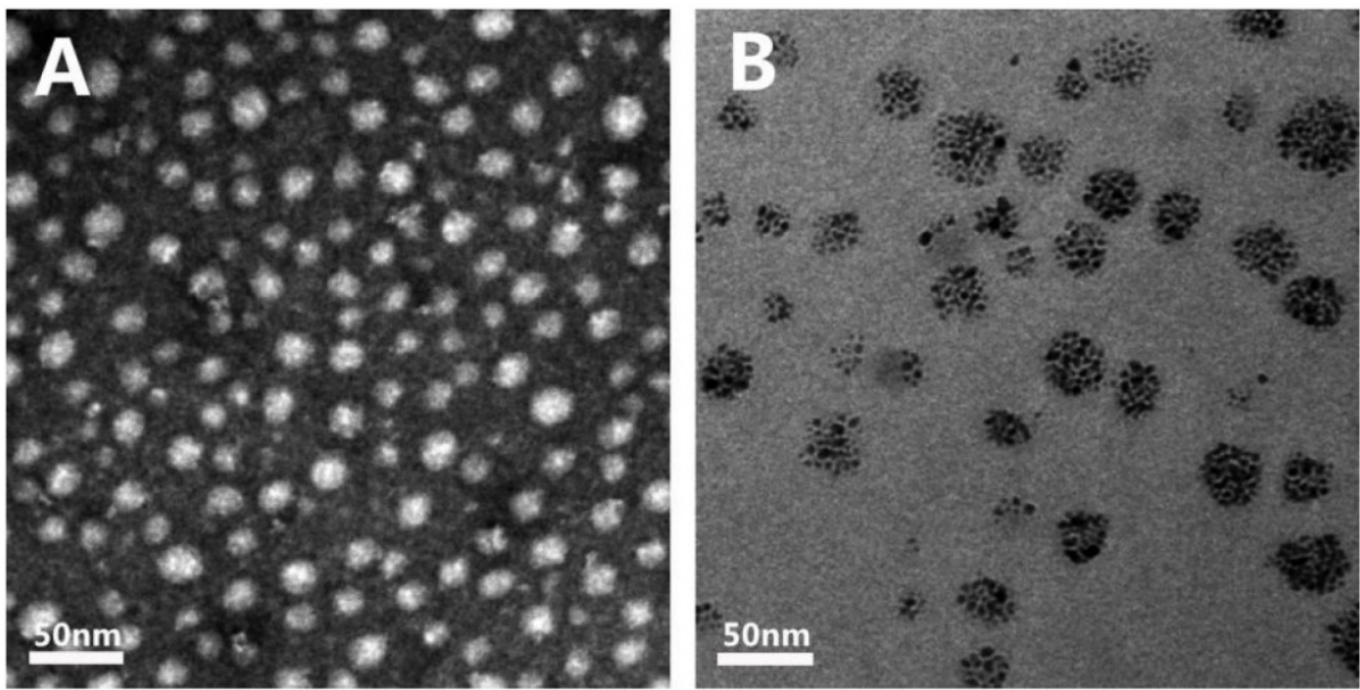

Figure 7. Transmission electron microscopy (TEM) images show the blank micelles (A) the mPEG-Lys3-CA4-NR/SPION micelles with a size of less than $40 \mathrm{~nm}$ and SPIONs loaded inside (B) Scale bars $=50 \mathrm{~nm}$.

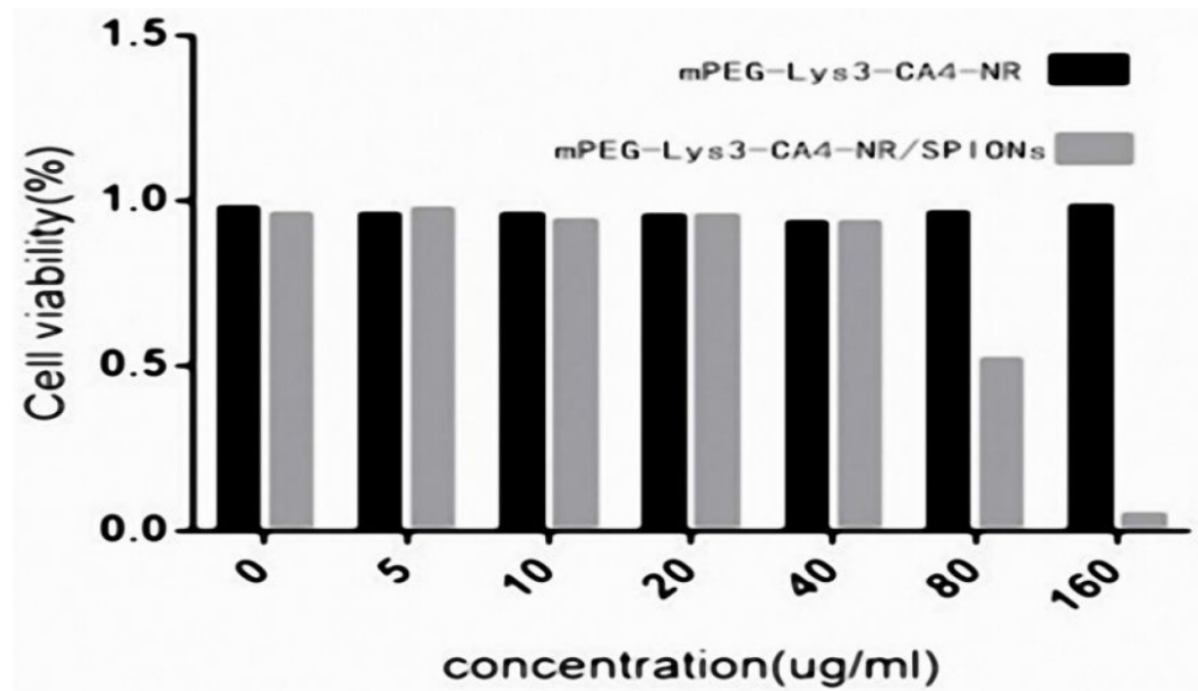

Figure 8. Cell viability of micelles in Raw264.7 cells as determined by the MTT assay after cells were incubated for $36 \mathrm{~h}(\mathrm{n}=3)$ with a series of concentrations of mPEG-Lys 3 -CA 4 - NR/SPION micelles and mPEG-Lys 3 -CA $A_{4}-N_{2}$ micelles, and the final concentration of iron of mPEG-Lys 3 -CA $A_{4}-\mathrm{NR} / \mathrm{SPION}$ micelleswas $0,5,10,20$, 40, 80, $160 \mu \mathrm{g} / \mathrm{mL}$ (the $\mathrm{mPEG}-\mathrm{Lys}_{3}-\mathrm{CA}_{4}-\mathrm{NR} / \mathrm{SPION}$ micelle and mPEG-Lys 3 -CA 4 -NR micelle concentrations were both 0, 108.8, 217.5, 435, 870, 1740, 3480 $\mu \mathrm{g} / \mathrm{mL}$. To highlight the iron, the $\mathrm{X}$-axis in this figure indicated the iron concentration). Notes: The data are represented as the means \pm standard deviations from 4 experiments; $P<0.05$

The Nile red fluorescence emission spectra for mPEG-Lys3-CA4-NR/SPION micelles are shown in Figure 9. As the incubation time was from $0.5 \mathrm{~h}$ up to $8 \mathrm{~h}$, the imaging indicated that the Nile red/SPIO co-loaded micelles were uptaken into cells, and the absorbed quantity was gradually increased when the incubation time was prolonged: the absorption showed a maximum value when the incubation time was $6 \mathrm{~h}$, and there were no significant changes when the incubated time was $8 \mathrm{~h}$ compared with that of $6 \mathrm{~h}$. The results indicated that the incubation time of $6 \mathrm{~h}$ was most favorable for cell labeling, and increasing the incubation time did not significantly improve the cellular uptake of mPEG-Lys3-CA4-NR/SPIO micelles. Otherwise, the cells incubated with mPEG-Lys3-CA4-NR/SPIO micelles showed very strong fluorescence, indicating that the micelles were effectively delivered into the Raw264.7 cells. Prussian blue staining was also performed to evaluate the cellular uptake ability of mPEG-Lys3-CA4-NR/SPIO micelles. As shown in Figure 10, it was also demonstrated that the micelles were effectively uptaken into the cells, and the absorbed quantity was gradually increased when the incubation time was prolonged and when the iron concentration was increased. 

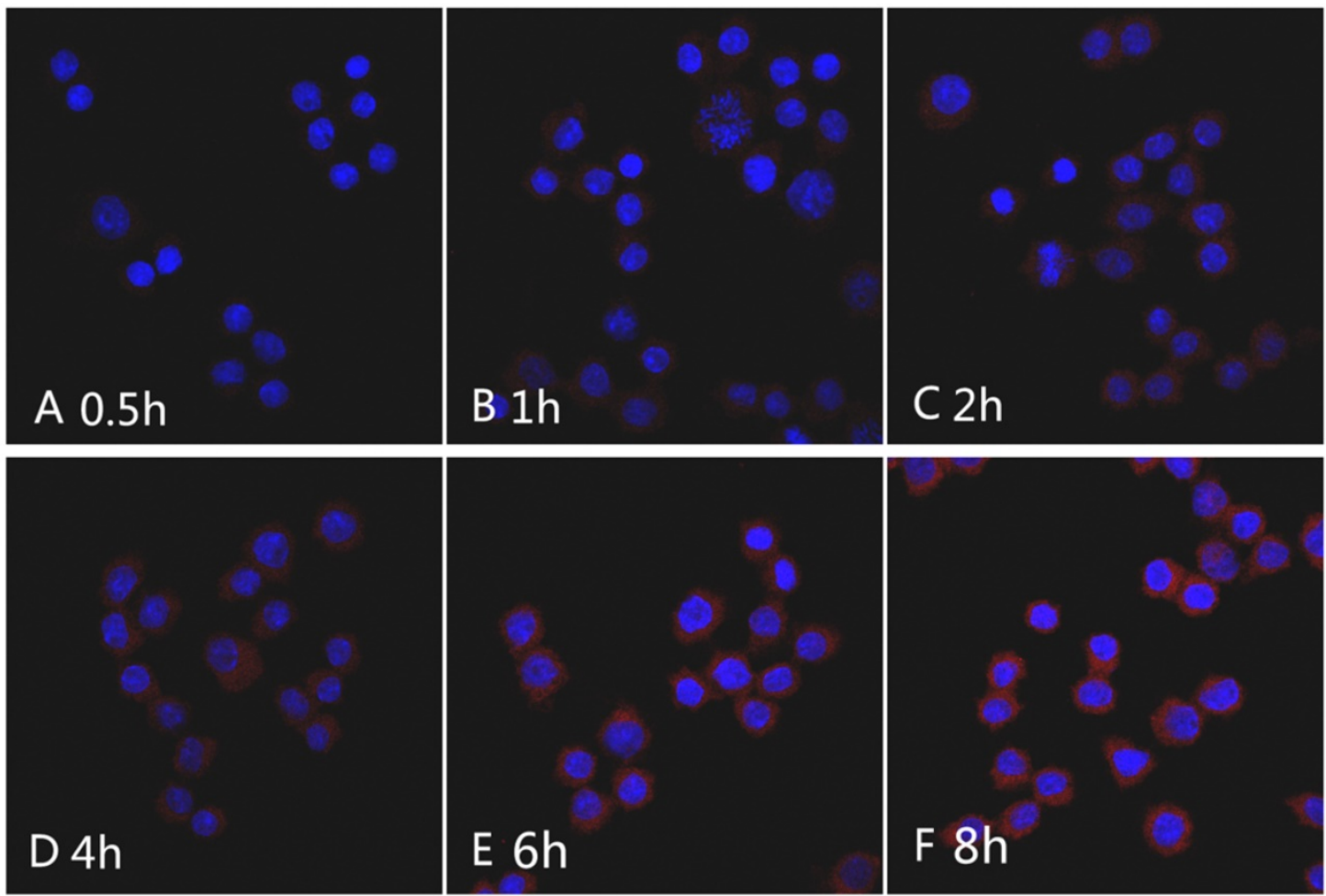

Figure 9. CLSM images of Raw264.7 cells incubated with the mPEG-Lys3-CA4-NR/SPION micelles for $0.5 \mathrm{~h}, 1 \mathrm{~h}, 2 \mathrm{~h}, 4 \mathrm{~h}, 6 \mathrm{~h}$, and 8 h. Nile red concentration: 1 $\mu \mathrm{g} / \mathrm{mL}$.

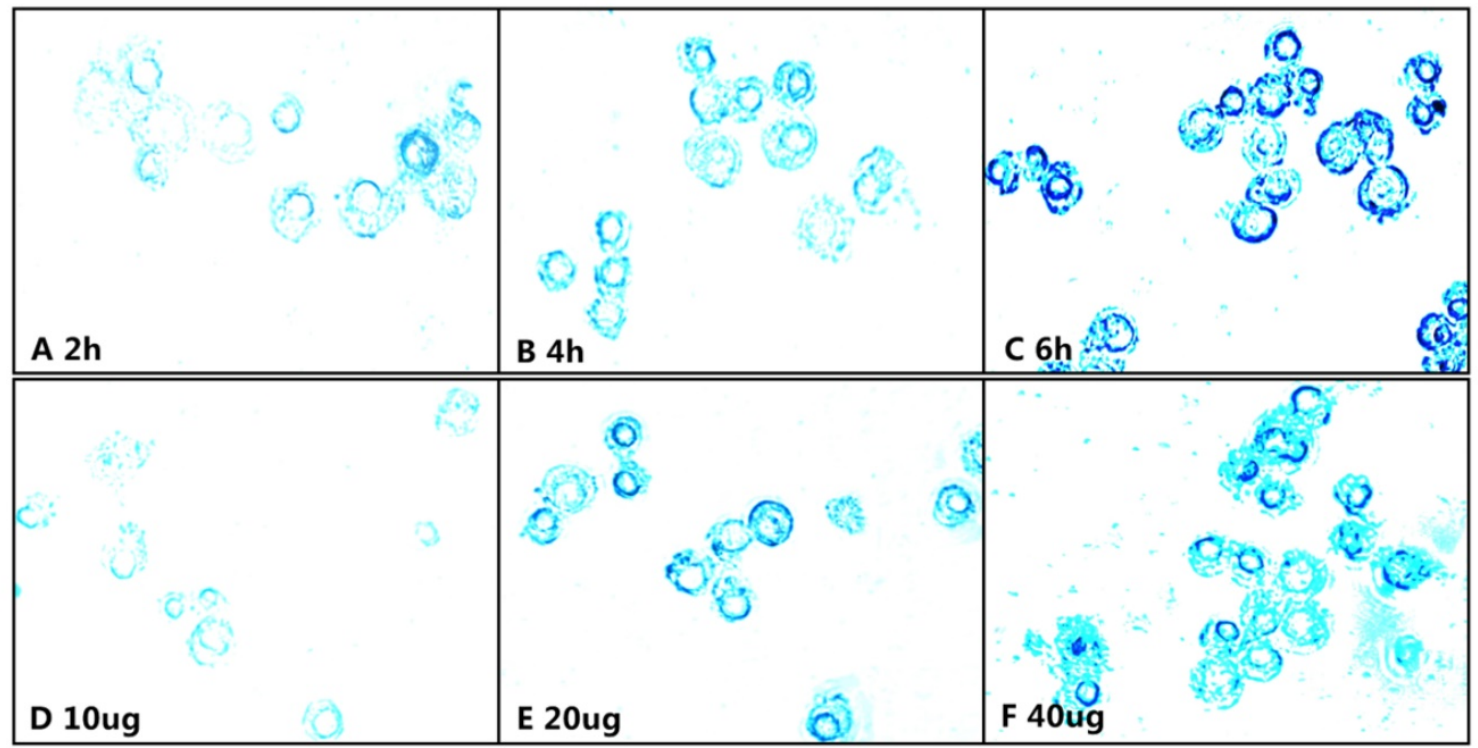

Figure 10. Prussian blue staining of the Raw264.3 cells incubated with the mPEG-Lys3-CA4-NR/SPION micelles, A-C show that Raw264.7 cells were incubated for 2,4 , and $6 \mathrm{~h}$ with an $\mathrm{Fe} 3+$ concentration of $40 \mu \mathrm{g} / \mathrm{mL}$; D-E show Raw264.7 cells inoculated with culture medium containing mPEG-Lys3-CA4-NR/SPION micelles with different $\mathrm{Fe} 3+$ concentrations of 10,20 , and $40 \mu \mathrm{g} / \mathrm{mL}$ for $6 \mathrm{~h}$.

\section{Magnetization and MRI sensitivity of mPEG-Lys3-CA4-NR/SPION micelles}

Based on the basic principle of MR imaging, the different content of water hydrogen nuclei in different organizations results in different image contrast along the longitudinal and transverse planes of the applied magnetic field. Consequently, like other MRI contrast agents that can increase the MRI signal intensity by shortening the hydrogen longitudinal relaxation time (T1) or decreasing the signal intensity by shortening the hydrogen transverse relaxation time (T2), as a superparamagnetic contrast agent, SPIO can significantly decrease the signal intensity by 
shortening the hydrogen transverse relaxation time (T2) and can cause darkening of the interfered regions. A normal lymph node with phagocytic function can take a substantial amount of contrast agent particles and, therefore, significantly reduce the T2 signal intensity of MRI. However, in the metastasis of lymph nodes, the macrophages are decreased due to the normal tissue being replaced by tumor cells [13, $20,21]$, the fewer macrophage cells, the less contrast agent uptake, which can therefore result in a decrease that maintains relatively high signal intensity. Based on this fact, our micelles of mPEG- Lys3- CA4NR/SPIONs can be used to better contrast between the diseased and healthy tissues. In our study, the transverse relaxivities $\mathrm{r} 2$ were calculated to determine the effect of the SPIO-polymer (mPEG- Lys3- CA4NR/SPIONs micelles) and hydrophilic SPIO, reflecting the ability of the SPIO-polymer (mPEGLys3- CA4- NR/SPION micelles) and hydrophilic SPIO to alter the T2 of water protons. Additionally, higher $\mathrm{r} 2$ leads to better effectiveness of a T2 agent.

As revealed in Figure 11, Figure 11A shows the T2-weighted imaging of mPEG-Lys3CA4-NR/SPION micelles (SPIO-polymer) and hydrophilic SPIO using phosphate-buffered saline (PBS) as the solvent at different Fe3+ concentrations as assessed on a 3.0T MRI scanner, and the MRI signal intensity of both was decreased significantly across the entire experiment with $\mathrm{Fe} 3+$ concentration ranging from 0 to $4 \mu \mathrm{g} / \mathrm{mL}$. In other words, the higher the concentration of $\mathrm{Fe} 3+$ is, the lower the MRI signal intensity will be. Furthermore, Figure 11B and Figure $11 \mathrm{C}$ indicated that, compared with the hydrophilic SPIO, mPEG-Lys3-CA4-NR/SPION micelles increase transversally(r 2), leading to a notably high $\mathrm{r} 2$ from $1.908 \mu \mathrm{g} / \mathrm{mL}^{-1} \mathrm{~S}^{-1}$ up to $5.032 \mu \mathrm{g} / \mathrm{mL}^{-1} \mathrm{~S}^{-1}$, making the mPEG-Lys3-CA4-NR/SPION micelles a highly sensitive MRI T2 contrast agent.

As shown in Figure 12, Figure 12A shows the T2-weighted imaging and T2 map of Raw264.7 cells inoculated with culture medium containing mPEG-Lys3-CA4-NR/SPION micelles and an Fe3+ concentration of $40 \mu \mathrm{g} / \mathrm{mL}$ for $0,0.5 \mathrm{~h}, 2 \mathrm{~h}, 4 \mathrm{~h}, 6 \mathrm{~h}$, and $8 \mathrm{~h}$, and the imaging was assessed on a 3.0T MRI scanner. The T2MI signal intensity was decreased when the incubation time was prolonged, and the T2 value had a minimum value when the incubation time was $6 \mathrm{~h}$ (Figure 12B); there were no marked changes when the incubation time was $8 \mathrm{~h}$ compared with that of $6 \mathrm{~h}$. The result was consistent with that previously reported. Figure $12 \mathrm{C}$ shows the T2 weighted imaging and T2 map of Raw264.7 cells inoculated with culture medium containing mPEG-Lys3-CA4-NR/SPION micelles with different $\mathrm{Fe} 3+$ concentrations of $0,5,10$, 20, $40 \mu \mathrm{g} / \mathrm{mL}$ for $6 \mathrm{~h}$. The results in Figure 12C and
Figure 12D indicated MRI T2 shortening with the increase in $\mathrm{Fe} 3+$ concentration. The signal reduction rates were $19.8 \%, 37.2 \%, 59.4 \%$, and $77.5 \%$ when the $\mathrm{Fe} 3+$ concentrations were 5, 10, 20, and $40 \mu \mathrm{g} / \mathrm{mL}$, respectively, compared with the blank control group.

MRI T2 shortening in the presence of mPEG-Lys3-CA4-NR/SPION micelles is closely related to several factors such as the Fe3+ concentration inside the core, diameter of the nanoparticles, surface charge, functional groups on the particle surface, and water-accessible surface area. Basically, MRI T2 shortening is associated with the increase in $\mathrm{Fe} 3+$ concentration. Additionally, recent reports have demonstrated that the $\mathrm{r} 2$ values can be increased by clustering SPIO agents within nanocontainers such as polymeric micelles [22] or liposomes [23]. In these cases, whatever the dilution factor applied to the solution, the local Fe3+ concentration is high and maintainable. Particularly, multiple magnetic nanoparticles encapsulated inside the hydrophobic core of one micelle can form a closed packing structure, resulting in much stronger T2 effects than micelles containing a single particle at the same iron concentration [24]. However, such a type of micellar encapsulation does not change the local Fe3+ concentration; thus, this may not be the major reason leading to the remarkably increased $r 2$ noted in the present study. Otherwise, when the particle size is decreased to the critical value, the agent changes from a multiple domain state to a single domain state - that is, the magnetic nanoparticle is similar to a giant paramagnetic atom and can respond fast to the external magnetic field, leading to high magnetic sensitivity. In addition, the phenomenon that r2 increases significantly may be explained by the large magnetic field heterogeneity around the nanoparticle through which water molecules diffuse.

To date, although several SPIO-related nanoparticle MRI contrast agents have been reported until now, the development of SPIO-related nanoparticles combined with a super small size and high MRI sensitivity is still receiving much attention. In our study, we have successfully synthesized mPEG-Lys3-CA4 micelles co-loaded with SPIO and Nile red into cores that combined relatively high MRI sensitivity and a super small particle size (less than 35 $\mathrm{nm})$. In a recent study, Qin et al. have reported a highly sensitive T2 contrast agent prepared by coating a single SPIO nanoparticle with Pluronic F127 copolymers [25]. However, in theirstudy, the nanoparticle diameter was approximately $70 \mathrm{~nm}$. The major limitation of our study is that the in vivo performance was not tested, and further follow-up of our study will be undertaken using in vivo experiment. 


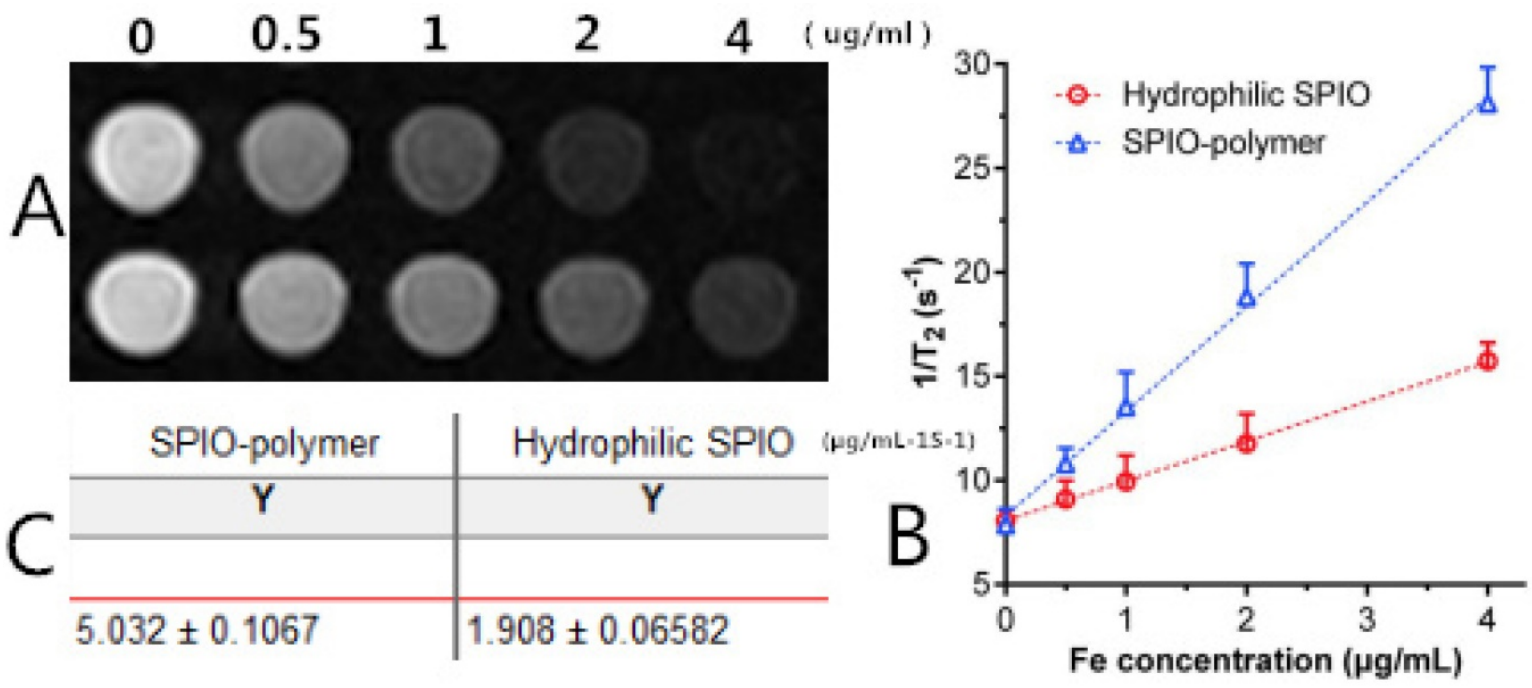

Figure 11. (A) T2-weighted imaging of mPEG-Lys3-CA4-NR/SPION micelle (SPIO polymer) solution and hydrophilic SPIO solution; (B) Relaxation rates 1/T2 of T2 as a function of iron concentration $(\mu \mathrm{g} / \mathrm{mL})$ for mPEG-Lys3-CA4-NR/SPION micelles ( $\mathrm{r} 2$ ) compared with hydrophilic SPIO; (C)T 2 relaxivities of mPEG-Lys3-CA4-NR/SPION micelles and hydrophilic SPIO.
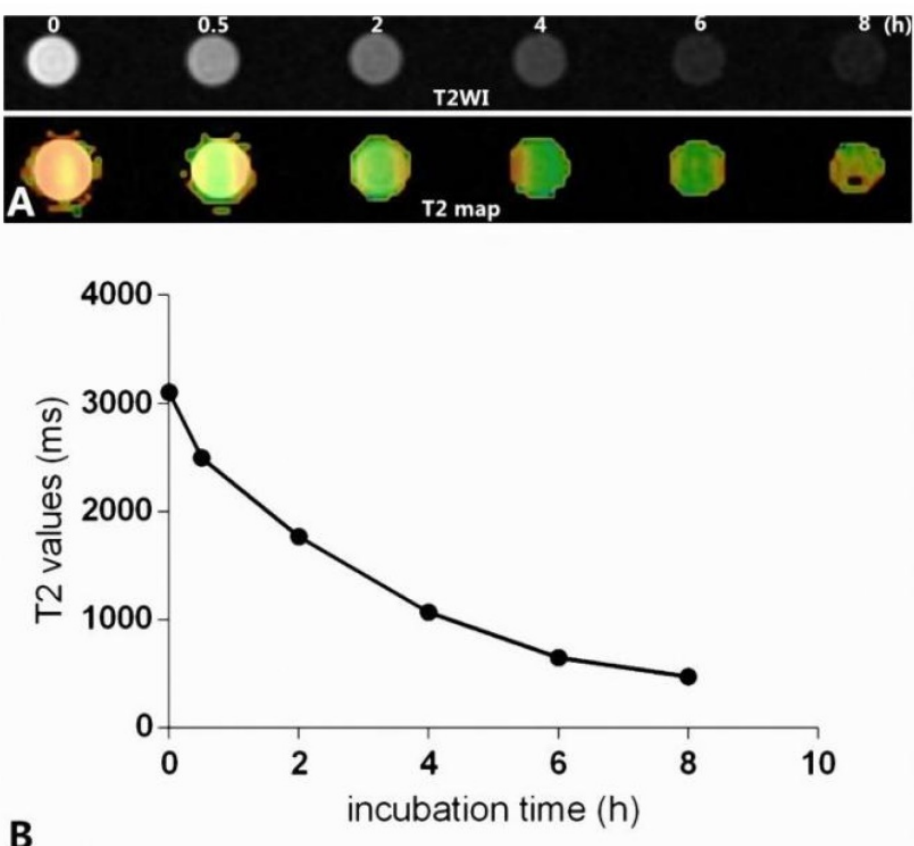

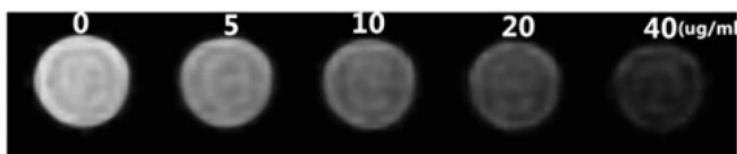

T2WI

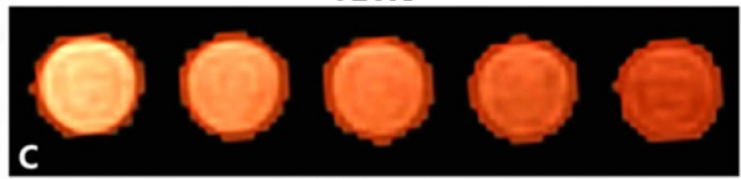

T2 map

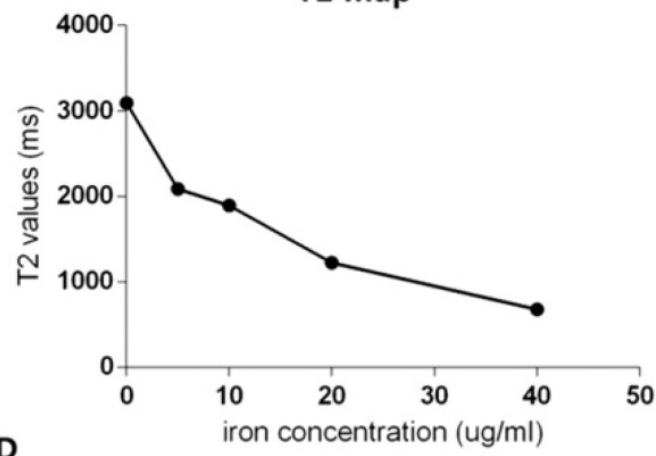

D

Figure 12. In vitro MR images. (A) shows that Raw264.7 cells were inoculated with culture medium containing mPEG-Lys3-CA4- NR/SPION micelles with an Fe3+ concentrations of $40 \mu \mathrm{g} / \mathrm{mL}$ for $0,0.5 \mathrm{~h}, 2 \mathrm{~h}, 4 \mathrm{~h}, 6 \mathrm{~h}, 8 \mathrm{~h}$ (T2Wl and T2 map); (C) shows Raw264.7 cells inoculated with culture medium containing mPEG-Lys3-CA4-NR/SPION micelles with different Fe3+ concentrations of 0, 5, 10, 20, and $40 \mu \mathrm{g} / \mathrm{mL}$ for $6 \mathrm{~h}$ (T2Wl and T2 map); (B)and (D) show the linear graph.

\section{Conclusions}

By loading hydrophobic superparamagnetic iron oxide nanoparticles (SPIONs) and Nile red into core micelles, we developed mPEG-Lys3-CA4-NR/SPION polymeric micelles with anultra-small size and high MRI sensitivity for MR and fluorescent bimodal lymphography, demonstrating a desirably super small size $(<40 \mathrm{~nm})$ and high MRI T2 sensitivity superior to that of hydrophilic SPIO. Our results revealed mPEG-Lys3-CA4-NE/SPIONs with high
MRI sensitivity and a super small particle size that are expected to provide a new strategy for the targeted therapy of lymph node metastasis and great theoretical research significance and clinical potentials. Further vivo study will be undertaken in future.

\section{Abbreviations}

MR: magnetic resonance; MRI: magnetic resonance imaging; SPIONs: superparamagnetic iron oxide nanoparticles; SPIO: superparamagnetic iron 
oxide; CLSM: Confocal Laser Scanning Microscopy; mPEG: cl-Methoxy-poly(ethylene glycol); mPEGLys3-CA4: mPEG-b-dendritic oligo-cholic acid; NR: nile red; TEM: Transmission electron microscopy; Boc-Lys(Boc)-OH: Di-tert-butoxycarbonyl-L-lysine; CA: Cholic acid; NMR: nuclear magnetic resonance; CDCl3: deuterochloroform; DMSO: dimethyl sulfoxide; TEM: Transmission electronic microscopy; MTT: methylthiazolyldiphenyl-tetrazolium bromide; CLSM: Confocal laser scanning microscopy.

\section{Acknowledgments}

This research was partly supported by the National Natural Science Foundation of China (81271561) and the Natural Science Foundation of Guangdong Province (2015A030313173).

\section{Competing Interests}

The authors have declared that no competing interest exists.

\section{References}

1. Okholm C, Svendsen LB, Achiam MP. Status and prognosis of lymph node metastasis in patients with cardia cancer -A systematic review. Surg Oncol. 2014; 23(3):140-146.

2. Mukesh G. Harisinghani, Saksena, Elena Brachtel, et al. MR Lymphangiography: Imaging Strategies to Optimize the Imaging of Lymph Nodes with Ferumoxtran-101.RadioGraphics. 2004; 24:867-878.

3. Quint LE, Bogot NR. Staging esophageal cancer. Cancer Imaging. 2008; 8 (Suppl A): 33-42.

4. Atula TS, Varpula MJ, Kurki TJ, et al. Assessment of cervical lymph node status in head and neck cancer patients: palpation, computed tomography and low field magnetic resonance imaging compared with ultrasound-guided fine-needle aspiration cytology. Eur J Radiol. 1997; 25:152-161.

5. Hilton $\mathrm{S}$, Herr HW, Teitcher JB, et al. CT detection of retroperitoneal lymphnode metastases in patients with clinical stage Itesticular nonseminomatous germ cell cancer: assessment of size and distribution criteria. AJR Am J Roentgenol. 1997; 169:521-525.

6. Anzai Y, Piccoli CW, Outwater EK, et al. Evaluation of neck and body metastases to nodes with ferumoxtran 10-enhanced MR imaging: phase III safety and efficacy study. Radiology. 2003; 228: 777-788.

7. Mansi A. Saksena, Anuradha Saokar, et al. Lymphotropic nanoparticle enhanced MR imaging(LNMRI) technique for lymph node imaging. European Journal of Radiology. 2006; 367-374.

8. Shiozawa M, Kobayashi S, Sato Y, et al. Magnetic resonance lymphography of sentinel lymph nodes in patients with breast cancer using superparamagnetic iron oxide: a feasibility study. Breast Cancer. 2014 Jul; 21(4):394-401.

9. Xu C, Miranda-Nieves D, Ankrum JA, et al. Tracking mesenchymal stem cells with iron oxide nanoparticle loaded poly(lactide-co-glycolide) microparticles. Nano Lett. 2012; 12:4131-4139.

10. Bull E, Madani SY, Sheth $\mathrm{R}$, et al. Stem cell tracking using iron oxide nanoparticles. Int J Nanomedicine. 2014; 9:1641-1653.

11. Neri M, Maderna C, Cavazzin C, et al. Efficient in vitro labeling of human neural precursor cells with superparama-gnetic iron oxide particles: Relevance for in vivo cell track-ing. Stem Cells. 2008; 26:505-516.

12. Weissleder R, Elizondo G, Wittenberg J, et al. Ultrasmall superparamagnetic iron oxide: an intravenous contrast agent for assessing lymph nodes with MR imaging. Radiology. 1990;175: 494-498.

13. Motomura $\mathrm{K}$, Ishitobi $\mathrm{M}$, Komoike $\mathrm{Y}$, et al. SPIO-enhanced magnetic resonance imaging for the detection of metastases in sentinel nodes localized by computed tomography lymphography in patients with breast cancer. Ann Surg Oncol. 2011;18 (12):3422-3429.

14. Wang YX, Hussain SM, Krestin GP. Superparamagnetic iron oxide contrast agents: physicochemical characteristics and applications in MR imaging. Eur Radiol. 2001; 11: 2319-2331.

15. Wang $\mathrm{Y}, \mathrm{Xiao} \mathrm{H}$, Fang $\mathrm{J}$, et al. Construction of negatively charged and environment-sensitive nanomedicine for tumor-targeted efficient siRNA delivery. Chem. Commun. 2016; 52:1194-1197.

16. Luo JT, Xiao K, Li YP, et al. Well-defined, size-tunable, multifunctional micelles for efficient paclitaxel delivery for cancer treatment. Bioconjugate Chem. 2010; 21:1216-1224.
17. Wen $\mathrm{XH}$, Wang $\mathrm{Y}$, Zhang $\mathrm{F}$, et al. In vivo monitoring of neural stem cells after transplantation in acute cerebral infarction with dual-modal MR imaging and optical imaging. Biomaterials. 2014;35: 4627-4635.

18. Torchilin VP, Papisov MI. Why do poly-ethylene glycol-coated liposomes circulate so long? J Liposome Res. 1994;4(3):725-739.

19. Kim DK, Mikhaylova M, Wang FH, et al. Starch-coated super-paramagnetic nanoparticles as MR contrast agents. Chem Mater. 2003; 15: 4343-4351.

20. Mizokami D, Kosuda S, Tomifuji M, et al. Superparamagnetic iron oxide-enhanced interstitial magnetic resonance lymphography to detect a sentinel lymph node in tongue cancer patients. Acta Otolaryngol. 2013; 133(4):418-423.

21. Ishiyama K, Motoyama S, Tomura N, et al. Visualization of lymphatic basin from the tumor using magnetic resonance lymphography with superparamagnetic iron oxide in patients with thoracic esophageal cancer. J Comput Assist Tomogr. 2006; 30 (2):270-275.

22. Nasongkla N, Bey E, Ren JM, et al. Multifunctional polymeric micelles as cancer-targeted, MRI-ultrasensitive drug delivery systems. Nano Letters. 2006; 6: 2427-2430.

23. Yuan JJ, Armes SP, Takabayashi Y, et al. Synthesis of biocompatible poly[2-(methacryloyloxy)ethyl phosphorylcholine]-coated magnetite nanoparticles. Lanomuir 2006; 22:10989-10993.

24. Ai H, Flask C,Weinberg B, et al. Magnetite-loaded polymeric micelles as ultrasensitive magnetic-resonance probes. Adv Mater. 2005; 17(16):1949-1952.

25. Qin J, Laurent S, Jo YS, et al. Coating nanocrystals with amphiphilic thermosensitive copolymers. Adv Mater. 2007; 19:1874-1874. 